\title{
Malignant Microangiopathy
}

\section{Dear Sir,}

In the controversy on basal membrane thickening of diabetes there is an aspect which I would like to stress and which in my opinion deserves careful attention.

There is no doubt that the thickening process progresses with the duration of the disease and after $15-20$ years $80 \%$ or more of the diabetics have a significant thickening of the basement membrane. It is clear, however, that the clinical course of diabetic microangiopathy is different from case to case and there is a consistent group of patients who after 20 years have no clinical evidence of eye or kidney disease. The majority of these patients have had a good control of the metabolic disturbance and in particular have been submitted to a three or four injections per day regimen with short acting insulins; or have been kept on strict diet even without any hypoglycaemic agents. Every experienced diabetologist can, however, testify that some of the patients without any clinical symptoms of microangiopathy have never been under strict control and not infrequently have had "brittle" diabetes for many years.

On the opposite side there are patients who develop the most severe consequences of microangiopathy even with good glycaemic control and apparently no therapeutic intervention so far can stop the progression of the disease. There is some suggestion that there is a genetic basis to such behaviour [1] which would account for at least some of the factors leading to such diabetic complications, although no definite conclusions can yet be drawn.

I want to stress, primarily, that some of these patients develop the most severe microangiopathy in the early stage of diabetes and progress rapidly to blindness and renal insufficiency. It would seem that some additional factor intervenes to give the disease a malignant character and for these cases the term of "malignant microangiopathy" would be appropriate.

The suspicion that immunological factors may play a role in such cases has brought us to study immune complexes in microangiopathy and we have found that a proportion of the patients who develop severe retinopathy within 3 years of the clinical onset of diabetes have increased immune complexes in the blood with highly significant differences when compared with matched controls $[2,3]$.

Our work suggests that in diabetics the presence of immune complexes is connected with the complications of diabetes and above all with the severe course of microangiopathy. So far no correlation has been found between immune complexes and metabolic control [4]. This increase of $\mathrm{AgAb}$ is observed both in insulin-treated diabetics and in patients kept on oral agents. This is in accordance with the finding that microangiopathy may develop in all diabetics without regard to the type of treatment.

In "malignant microangiopathy" immunological factors may be superimposed on metabolic factors and play a major role in the pathogenesis of the condition.

Much work remains to be done to test this hypothesis. The genetic background of such cases must be more clearly defined, and the factors which facilitate the increase of immune complexes in blood must also be clarified; moreover the antigens of such immune complexes have to be identified.

\section{Domenico Andreani}

\section{References}

1. Cudworth A (1978) The HLA system, autoimmune endocrinology and diabetes mellitus. Eur J Clin Invest 8: 355-357

2. Irvine WJ, Di Mario U, Guy K, Javicoli M, Pozzilli P, Lumbroso B, Andreani D (1978) Immune complexes in diabetic microangiopathy. $\mathrm{J}$ Clin Lab Immunol 1: 187

3. Andreani D, Di Mario U, Javicoli M, Pozzilli P, Lumbroso B, Guy K, Irvine WJ (1978) Immune complexes and diabetic retinopathy. Diabetologia 14: 213

4. Di Mario U, Javicoli M, Ventriglia L, Galfo C, Bellagamba C, Trionfera $\mathrm{G}$, Cicconetti $\mathrm{C}$, Andreani $\mathrm{D}$ (in press) Immune complexes, microangiopathy and metabolic control in diabetes. Proc. 10th IDF Congress, Vienna, Sept. 9-11, 1979

Domenico Andreani

Professor of Endocrinology

University of Rome

Policlinico Umberto I

I-00100 Rome

Italy 